\title{
Corrigendum to "Modelling wildland fire propagation by tracking random fronts" published in Nat. Hazards Earth Syst. Sci., 14, 2249-2263, 2014
}

G. Pagnini ${ }^{1,2}$ and A. Mentrelli ${ }^{1,3}$

${ }^{1}$ BCAM - Basque Center for Applied Mathematics, Alameda de Mazarredo 14, 48009 Bilbao, Basque Country, Spain ${ }^{2}$ Ikerbasque, Basque Foundation for Science, Alameda Urquijo 36-5, Plaza Bizkaia, 48011 Bilbao, Basque Country, Spain ${ }^{3}$ Department of Mathematics \& Alma Mater Research Center on Applied Mathematics (AM) ${ }^{2}$, University of Bologna, via Saragozza 8, 40123 Bologna, Italy

Correspondence to: G. Pagnini (gpagnini@bcamath.org)

We would like to inform you that the third affiliation in the published manuscript is wrong. You will find the correct affiliation above. 